\title{
Primary laryngeal localization of multiple myeloma: A case report
}

\author{
EUGENIA ALLEGRA ${ }^{1}$, NICOLÒ MARINO ${ }^{2}$, DOMENICO MODICA ${ }^{2}$, \\ CARMELA EMMANUELE ${ }^{3}$ and VINCENZO SAITA ${ }^{2}$
}

\author{
${ }^{1}$ Department of Health Sciences-Otolaryngology, School of Medicine, 'Magna Graecia' University of Catanzaro, \\ I-88100 Catanzaro; Departments of ${ }^{2}$ Otolaryngology and ${ }^{3}$ Histopathology, 'Cannizzaro' Hospital, I-95100 Catania, Italy
}

Received May 16,2016; Accepted November 15, 2016

DOI: $10.3892 / \mathrm{mco} .2017 .1122$

\begin{abstract}
Multiple myeloma is a lymphoproliferative disease that may involve the bone marrow as well as extramedullary soft tissues. However, laryngeal localization of multiple myeloma is extremely rare. We herein present the case of a 68 -year-old male patient with a history of dyspnea, dysphonia and dysphagia. Laryngoscopic examination revealed a lesion involving the right glottis and right vestibular (false) vocal fold, with absence of ipsilateral laryngeal motility and constriction of the airway. Computed tomography and magnetic resonance imaging revealed a gross swelling infiltrating the right glottis and right false vocal fold, sized $33 \times 19 \times 33 \mathrm{~mm}$, with sub-centimeter laterocervical lymph nodes bilaterally. Careful integration of the clinical manifestations with the radiological and pathological data led to the diagnosis of multiple myeloma. Given the rarity of this localization, the purpose of this study was to increase knowledge of this disease among ear, nose and throat specialists, in order to enable a more timely diagnosis.
\end{abstract}

\section{Introduction}

Multiple myeloma (MM) is a neoplastic disease characterized by the proliferation and accumulation of B lymphocytes and plasma cells that synthesize monoclonal immunoglobulin (M component). Monoclonal plasma cell neoplasms include MM, solitary plasmacytoma, Waldenström's macroglobulinemia, and gammapathies associated with lymphoproliferative syndromes.

In $95 \%$ of the cases, $\mathrm{MM}$ is demonstrable with an increase of the $\mathrm{M}$ component in the serum and/or in the urine $(1,2)$. The disease may involve the bone marrow as well as soft tissues; its primary location is usually represented by a bone marrow hematopoietic content area, with secondary involvement of an extramedullary site, which is often represented by the

Correspondence to: Professor Eugenia Allegra, Department of Health Sciences-Otolaryngology, School of Medicine, 'Magna Graecia' University of Catanzaro, Viale Europa, Germaneto, I-88100 Catanzaro, Italy

E-mail: eualle@unicz.it

Key words: multiple myeloma, larynx, extramedullary myeloma upper aerodigestive tract (3). Primary localization in an extramedullary site is less frequent, isolated, or associated with a secondary location in the bone marrow. MM with a laryngeal localization represents $1 \%$ of the malignancies that affect this organ and predominantly affects men between the sixth and seventh decades of life, with a male:female ratio of 2:1 (1).

\section{Case report}

A 68-year-old male non-smoker with a clinical history of dyspnea, dysphonia and dysphagia, exhibited worsening of his condition 4 months prior to seeking medical advice. Laryngoscopic examination revealed a lesion involving the right glottis and right vestibular (false) vocal fold, with absence of ipsilateral laryngeal motility and constriction of the airway (Fig. 1).

Computed tomography (CT) and magnetic resonance imaging (MRI) revealed a gross swelling infiltrating the

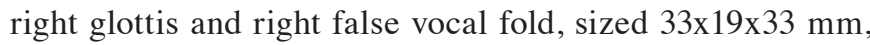
with sub-centimeter laterocervical lymph nodes bilaterally (Figs. 2 and 3). A whole-body CT revealed no pathological findings. For diagnostic purposes and to resolve the severe dyspneic symptoms, tracheostomy with targeted biopsies (later found to be negative for malignancy) was performed in July 2015. Due to the persistence of the lesion, which was refractory to treatment with anti-inflammatory drugs and antibiotics, new biopsies were collected during direct laryngoscopy after 1 month; the subsequent histological examination revealed a 'benign laryngeal mucosa, infiltrated by lymphocytes, plasma cells and granulocytes, a lamina propria rich in mixed glands (mostly mucous) and excretory ducts, and striated muscle'. Given the inconclusive diagnosis, further biochemical, clinical and immunohematological tests were performed and the results were as follows: Negative for Bence Jones protein; increased $\alpha 2$ and $\beta 1$ paraproteins (by 13.90 and $25.50 \%$, respectively); and decreased albumin, $\gamma$-globulin and $\mathrm{A} / \mathrm{G}$ ratio (by 42.90 , $7.60 \%$ and 0.75 , respectively) on the proteinogram. IgAк was found in the subsequent immunofixation, also confirmed by immunohistochemistry of a bioptic specimen that was positive for CD138 (Fig. 4).

The bone marrow contained $40 \%$ plasma cells, which were also positive for CD138. Staging by positron emission tomography/CT imaging, performed 1 month after whole-body $\mathrm{CT}$, revealed localization of the disease in the $\mathrm{V}$ right rib. At the end of the diagnostic process (September 2015) the 


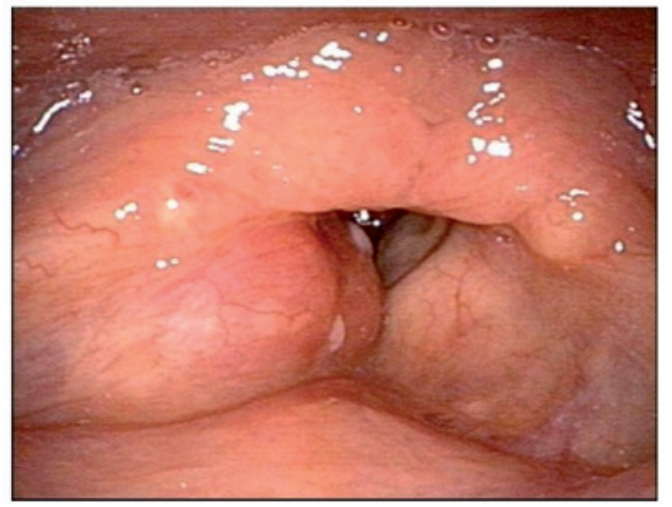

Figure 1. Endoscopic image of the larynx prior to treatment.



Figure 2. Computed tomography image showing a gross swelling infiltrating the right glottis and right false vocal fold.

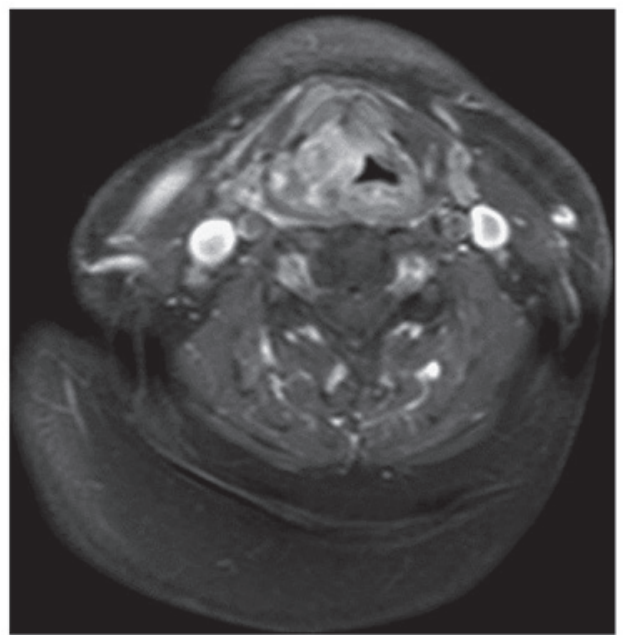

Figure 3. Magnetic resonance imaging showing a gross swelling infiltrating the right glottis and right false vocal fold.

patient underwent 6 cycles of chemotherapy with bortezomib (Velcade), thalidomide and dexamethasone (VTD scheme), achieving partial remission of the disease. At the end of

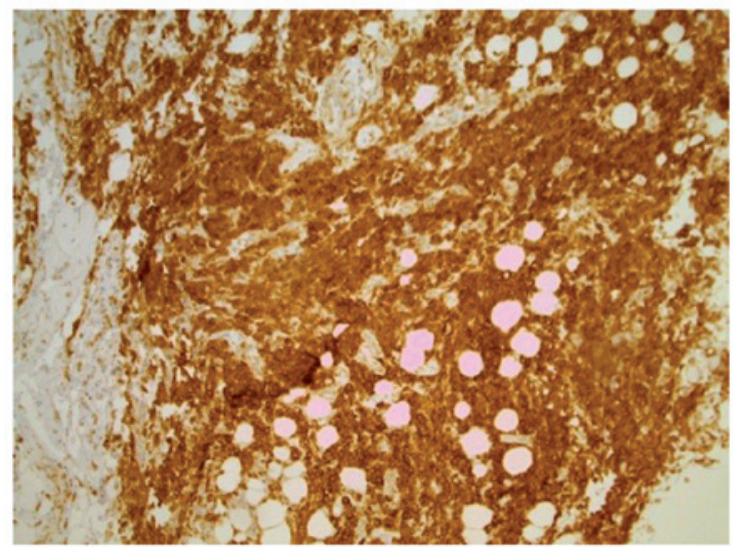

Figure 4. Immunohistochemical staining showing diffuse expression of CD138 by the infiltrating lymphocytes, plasma cells and granulocytes.



Figure 5. Endoscopic image of the larynx at the end of 6 cycles of chemotherapy.

the chemotherapy (March 2016), an endoscopic examination (Fig. 5) and MRI control showed a reduction in the dimensions of the pathological tissue $(20 \times 10 \times 12 \mathrm{~mm})$, which infiltrated the right false vocal fold and glottis and extended to the anterior commissure, with right persistent disease in the right paraglottic space and signal alteration of the thyroid cartilage. The relief of the airway constriction allowed for decannulation of the patient in April 2016, after a median follow-up of 6 months. The patient is currently on maintenance treatment with interferon and steroids and the next laryngoscopic control is scheduled for January 2017.

\section{Discussion}

Laryngeal localization of MM is extremely rare as a primary or secondary site to a primary marrow localization, which is a more frequent occurrence. The etiology is not known. Point mutations of the N-ras and K-ras genes, as well as mutations or deletions of the tumor suppressor genes p53 and Rb-1, are delayed molecular events that may play a role in the onset and progression of this disease (1). In decreasing order of frequency, the most commonly affected laryngeal areas are the epiglottis, glottis, false vocal folds, aryepiglottic folds and thyroid cartilage (3). The disease rarely presents with a sudden onset. Dyspnea, dysphonia and dysphagia are correlated with 
disease localization $(2,4)$. The lesion may present as a polypoid mass or swelling involving the entire organ, with a smooth, benign mucosal surface. The differential histological diagnosis may be challenging, as plasma cell infiltrates may also be encountered in various benign conditions, such as chronic inflammatory diseases, inflammatory polyps and amyloid deposits $(5,6)$. Therefore, histological diagnosis should be supported by hematological and radiological examination. The blood count in MM patients may reveal anemia and/or leukopenia and/or thrombocytopenia. In 95\% of the MM cases, electrophoresis and immunofixation indicate the presence of an $\mathrm{M}$ component. A urine test is only useful for detecting light chains (Bence Jones protein). Bone marrow biopsy shows an excess of plasma cells ( $>10 \%$ of the total nucleated cell population), which may extend to total replacement of the normal myeloid parenchyma. Immunohistochemistry is crucial for determining positivity of B lymphocytes and plasma cells for the CD138 marker in the bone marrow as well as in extramedullary sites $(3,5)$. For prognostic purposes, the determination of the serum concentration of b2-microglobulin is relevant (1). The overall clinical assessment of the patient applies diagnostic imaging (chest X-ray and whole-body CT and MRI), which is useful in determining the possible presence of the disease in other locations and in evaluating lymph node stations. The therapy mainly includes radiation and/or chemotherapy, with subsequent transplantation of autologous or allogeneic stem cells $(1,7)$.
Despite its low incidence, primary laryngeal localization of MM should always be considered in the presence of a laryngeal mass of undetermined clinical nature.

\section{References}

1. McKenna RW, Kyle RA, Kuehl WM, Grogan TM, Harris NL and Coupland RW: Plasma cell neoplasms. In: WHO Classification of Tumours of Haematopoietic and Lymphoid Tissues. Swerdlow SH, Campo E, Harris NL, Jaffe ES, Pileri SA, Stein H, Thiele J and Vardiman JW (eds). Vol 2. IARC, Lyon, pp200-213, 2008.

2. Nofsinger YC, Mirza N, Rowan PT, Lanza D and Weinstein G: Head and neck manifestations of plasma cell neoplasms. Laryngoscope 107: 741-746, 1997.

3. Mitchell HK, Garas G, Mazarakis N and McGlashan J: Extramedullary relapse of multiple myeloma in the thyroid cartilage. BMJ Case Rep 2013: pii.bcr2013200689, 2013.

4. Nampoothiri MP, Kumar KP and Sajina VK: Multiple myeloma presenting as stridor: A case report. Indian J Otolaryngol Head Neck Surg 58: 111-112, 2006.

5. Grobman AB, Vivero RJ, Campuzano-Zuluaga G, Ganjei-Azar P and Rosow DE: Laryngeal involvement of multiple myeloma. Case Rep Oncol Med 2012: 257814, 2012.

6. Aslan I, Yenice H and Baserer N: An indolent course of multiple myeloma mimicking a solitary thyroid cartilage plasmacytoma. Eur Arch Otorhinolaryngol 259: 84-86, 2002.

7. Michalaki VJ, Hall J, Henk JM, Nutting CM and Harrington KJ: Definitive radiotherapy for extramedullary plasmacytomas of the head and neck. Br J Of Radiology 76: 738-741, 2003. 